\title{
ASSISTÊNCIA DE ENFERMAGEM À CRIANÇA HOSPITALIZADA: breve retrospectiva
}

Maria Cristina Frères de Souza* Isabel Cristina dos Santos Oliveira**

\section{Resumo}

O estudo enfoca o panorama da política de saúde infantil, em destaque no contexto hospitalar, na cidade do Rio de Janeiro, tendo como propósito descrever a assistência de enfermagem à criança hospitalizada na primeira metade do século. Trata-se de um estudo histórico, cujas fontes primárias escritas correspondem aos artigos publicados na Revista Brasileira de Enfermagem no período em estudo. As fontes secundárias estão constituídas pela literatura pertinente à temática. No início do século $X X$, o setor saúde deixa o caráter social e filantrópico para ser subsidiado pelo governo. Em relação a assistência à criança, inúmeros hospitais são inaugurados nos anos 30 e 40, apesar da política nacional de saúde caracterizar-se por ações preventivas. Conclui-se que a assistência à criança até os anos 50 caracteriza-se por transformações técnico-científicas no cenário hospitalar, favorecendo a construção do saber da enfermagem na área de pediatria.

Descritores: enfermagem; política de saúde; criança hospitalizada; história

\begin{abstract}
This study focuses on the overview of the childhood health policy, highlighted in the context of a hospital, in the city of Rio de Janeiro, aiming at describing the nursing assistance provided to children in hospitals in the first half of the century. It is a historical study, the primary written sources of which correspond to articles published in the Revista Brasileira de Enfermagem during the period under scrutiny. The secondary sources are made up of literature relevant to the topic. In the beginning of the 20th century, health care loses its social and philanthropic character to be subsidized by the government. Concerning childhood assistance, numerous hospitals opened in the 30's and 40's, even though the national healthcare policy was based on preventive actions. It may be concluded that childhood assistance up to the 50's was characterized by technical and scientific transformations in the hospital scenario, encouraging the construction of nursing knowledge in the area of paediatrics. Descriptors: nursing; health policy; hospitalised child; history

Title: Nursing care of child: short retrospection
\end{abstract}

\section{Resumen}

El estudio enfoca el panorama de la política de la salud infantil, en el contexto hospitalario en Rio de Janeiro. El propósito es describir la asistencia de enfermería a los niños en los hospitales. Es un estudio histórico, cuyas fuentes primarias escritas corresponden a los artículos publicados en la Revista Brasileña de Enfermería durante la primera mitad del siglo. Las fuentes secundarias se constituyen por la literatura sobre esa temática. A principios del siglo XX, el sector de la salud deja de tener un carácter social y filantrópico y pasa a ser subvencionado por el gobierno. Respecto a la asistencia infantil, innumerables hospitales se inauguran en los años 30 y 40, a pesar de que la política nacional de salud se caracterizaba por acciones preventivas. Se concluye que la asistencia a los niños hasta los años 50 está caracterizada por unas transformaciones técnico-científicas en el escenario de los hospitales y con ello favorece la construcción del saber de enfermería en el campo de la pediatría.

Descriptores: enfermería; política de la salud; niños ingresados; historia

Titulo: Asistencia de enfermería al niño: breve retrospectiva

\section{Introdução}

Este estudo enfoca o panorama da política de saúde infantil, em destaque, no contexto hospitalar na cidade do Rio de Janeiro, tendo como propósito descrever a assistência de enfermagem à criança hospitalizada na primeira metade do século $X X$.

Considero importante apresentar a assistência na área de saúde dentro dos hospitais gerais e pediátricos, para um melhor entendimento dos fatores intrínsecos e extrínsecos que influenciaram na assistência à criança hospitalizada e a prática da enfermagem.

Trata-se de um estudo histórico. As fontes primárias escritas são os artigos publicados na revista Brasileira de Enfermagem no período em estudo. As fontes secundárias estão constituídas pela literatura pertinente à temática.

\section{Os marcos históricos da assistência de enfermagem à criança hospitalizada}

A assistência à criança estava vinculada diretamente ao contexto histórico-social e econômico do país. No início do século $X X$, ocorreram mudanças no setor da saúde, "caracterizou-se por um período de transição quanto à assistência social e filantrópica em favor de uma assistência à saúde com subvenção particular e/ou pública"(1: 25).

Em 1901, foi inaugurado no Rio de Janeiro, o Instituto de Proteção e Assistência à Infância, fundado por Carlos Artur
Moncorvo Filho. Esta instituição teve ampla repercussão, sendo reconhecida em nível municipal (Decreto n. 139 - 17/11/1909) e federal (Decreto n. 3877 - 12/11/1919), com instalações de filiais por todo o país, cujos objetivos eram atender à criança pobre nos múltiplos problemas psico-patológicos ${ }^{(2 ; 3)}$.

Foi inaugurada, em 1910, a Policlínica das Crianças, mantida pela Santa Casa de Misericórdia. O primeiro diretor foi o médico Antonio Fernandes Figueira, que fundou a Sociedade Brasileira de Pediatria no mesmo ano. A partir de 1918, a Irmandade da Santa Casa de Misericórdia do Rio de Janeiro manteve também o Hospital São Zacarias, que alojou uma clínica pediátrica(4)

Na década de 20, ocorreu a Reforma Carlos Chagas, reorganizando os serviços de saúde pública, sendo criado o Departamento Nacional de Saúde Pública (DNSP) vinculado ao Ministério da Justiça e Negócios Interiores, com ação em várias áreas, incluindo a higiene infantil e supervisão dos hospitais públicos federais ${ }^{(5)}$.

Em 1921, com o auxílio e cooperação do governo dos Estados Unidos e financiamento da Fundação Rockefeller, acontece a fundação da Escola de Enfermeiras do DNSP (posteriormente Escola de Enfermagem Anna Nery) moldada ao sistema de Florence Nightingale, originário da Inglaterra, que chega ao Brasil proveniente dos Estados Unidos, implantando a enfermagem profissional(6). As enfermeiras realizavam orientações às mães, nos consultórios de higiene

\footnotetext{
* Enfermeira gerente do setor de Pediatria Oncológico do Instituto Nacional de Câncer / RJ. Mestre em Enfermagem pela Escola de Enfermagem Anna Nery / UFRJ. Membro do Núcleo de Pesquisa de História da Enfermagem Brasileira (Nuphebras) da EEAN / UFRJ. ***Enfermeira. Doutora em Enfermagem. Professora Adjunta do Departamento de Enfermagem Médico-Cirúrgica - EEAN/UFRJ. Pesquisadora / CNPq.

E-mail do autor: constanciar@ig.com.br
} 
infantil, sobre: cuidados da gestação, parto, pós-parto e com seus filhos, com o objetivo de combater a mortalidade infantil(7).

Com a destruição da hegemonia cafeeira na década de 30, a política de saúde toma novas direções. Uma nova aliança no poder é formada com a revolução de 30 , e a burguesia industrial nascente, os cafeicultores, os grandes comerciantes importadores e as oligarquias dissidentes coexistiram de forma instável(8)

A política de saúde de caráter nacional centralizou-se, a partir de 1930, em dois subsetores: saúde pública e medicina previdenciária, prevalecendo o de saúde pública ${ }^{(9)}$. Enquanto, com a evolução do ensino da pediatria, a assistência à criança foi institucionalizada com dois tipos de prática médica: puericultura e assistência médica privada ${ }^{(10)}$.

$\mathrm{Na}$ época, a política social se fundamentava no assistencialismo de caráter populista como base para difusão de princípios político-ideológicos, como cidadania, direitos sociais e nacionalidade ${ }^{(11)}$.

O Presidente Getúlio Vargas iniciou a construção de hospitais da Prefeitura do Distrito Federal, como o Hospital Jesus especializado em clínicas médica e cirúrgica infantis ${ }^{(12: 217-}$ 219) e o Instituto de Puericultura no âmbito federal(13).

Cabe destacar que o Hospital Jesus foi inaugurado em 20/6/1935, passando a denominar - se Hospital Estadual Jesus (artigo 102 do Decreto n. 66 -18/9/63); e o Instituto de Puericultura era um órgão assistencial e de ensino da puericultura e pediatria, incorporado à Universidade do Brasil (Decreto - lei n. 98 - 23/ 12/37) e denominado Instituto de Puericultura e Pediatria Martagão Gesteira da UFRJ em 1954.

O Presidente reconhecia a necessidade de uma rede (estados, municípios e iniciativa privada) eficiente de serviços de amparo à maternidade e à infância através de um discurso populista apoiado nas idéias eugênicas, provenientes do nazismo alemão, de melhorar a raça e dar ao país gente forte e sadio $^{(11)}$

A participação político-social das enfermeiras durante o Estado Novo estava voltada para o aspecto social das doenças nas crianças, destacando a importância de uma infância sadia $^{(14)}$.

Em seus artigos, as enfermeiras destacavam o alto índice de mortalidade infantil e de analfabetismo, mas a relação entre as condições sócio-econômicas e a saúde da população, não eram discutidas. Também não apresentavam análise crítica, reproduzindo os discursos ideológico-governamentais ${ }^{(1)}$.

Durante a década de 40, persiste o movimento eugênico através das metas governamentais e nos discursos dos puericultores, refletindo na produção científica das enfermeiras ${ }^{(1)}$.

O médico sanitarista João de Barros Barreto realiza uma reforma no Departamento Nacional de Saúde (DNS) em 1941, durante o Estado Novo. Com esta organização, tentou-se coordenar as atividades de saúde em âmbito federal e dedicar recursos à assistência à maternidade e à infância(5).

A construção de hospitais infantis ou serviço clínico infantil em pavilhões anexos aos hospitais gerais era uma necessidade para atender às crianças doentes, através de estabelecimentos adequados ${ }^{(15)}$.

A conjuntura sócio-econômica favoreceu o crescimento dos ideais redemocratizantes. A hegemonia político-econômica norte-americana limita a expansão da política econômica nacionalista praticada pelo Governo Vargas. Todos estes fatores contribuíram para a queda de Getúlio Vargas, em 1945, assumindo o poder através de eleições diretas, o general Eurico Gaspar Dutra(16)

Conforme a citada autora, o setor químico e farmacêutico encontrava-se entre as indústrias que mais se expandiram no período de 1939 a 1952. A produção farmacêutica internacional realizou a descoberta de novos medicamentos e antibióticos.

No pós-guerra, no setor saúde surge importantes mudanças, tais como o surgimento de órgãos internacionais como: Organização Mundial da Saúde (OMS) e Fundo Internacional de Socorro à Infância (FISI) ou Fundo das Nações Unidas para a Infância (UNICEF); e com a venda de implementos terapêuticos surge a hegemonia da influência curativa $^{(17)}$.

Nos fins da década de 40, construiu-se no Rio de Janeiro, três hospitais para atender à clientela infantil. O Hospital Infantil inaugurado em 1947, para atendimento da clínica pediátrica geral e passando a denominar-se Hospital Estadual Salles Netto em 1965. Em 1948, inaugurou-se o Hospital Barata Ribeiro com o objetivo de atender os alunos matriculados nos estabelecimentos de ensino de $1^{\circ}$ grau da Prefeitura e particulares, nas clínicas cirúrgica e ortopédica. Este hospital passou a atender também a clientela adulta, em 1955, motivada pela obra de Hospital Souza Aguiar, recebendo os pacientes e funcionários do referido hospital. Por iniciativa do Ministério da Aeronáutica, inicia-se, em 1949, a construção do Centro MédicoPedagógico Nossa Senhora do Loreto sendo inaugurado em 1950, destinado ao tratamento e internação de crianças portadoras de afecções cirúrgicas. A partir de 1963, este centro denomina-se Hospital Estadual Nossa Senhora do Loreto ${ }^{(12)}$.

Em 1950, durante o governo Dutra, amplia-se a assistência médica aos segurados da previdência social, ocorre a compra e construções de hospitais e aquisição de equipamentos médicos ${ }^{(18)}$

A dicotomia aumenta em relação aos aspectos preventivos e curativos, ficando a prática sanitária em segundo plano. Na área de pediatria, a assistência à criança concentrase nos hospitais, com as especializações, surgindo as clínicas pediátricas. Com isso, é sentida a necessidade de maior demanda de pessoal de enfermagem mais qualificado nos hospitais. Neste sentido, a Associação Brasileira de Enfermagem (criada em 1926 com a denominação de Associação Brasileira de Enfermeiras diplomadas) e agências internacionais como a OMS, objetivando a capacitação de pessoal, ressaltam a necessidade de criação de escolas de Enfermagem ${ }^{(10)}$

Segundo Oliveira(1:52), na década de 50, o hospital assumiu uma posição central na prestação de saúde, concentrando diversas especialidades, e também, apoiado na maior utilização de medicamentos, emprego mais intensivo de equipamentos e serviços de diagnósticos.

As enfermeiras destacavam o aprendizado contínuo em diferentes áreas, como uma necessidade para atualização acerca do desenvolvimento científico e tecnológico ${ }^{(19)}$.

Veloso(20), em seu artigo descreve a necessidade de criação de hospitais pediátricos ou enfermarias específicas nos hospitais gerais, inclusive com a participação da enfermagem no plano de construção ou adaptação, ressaltando a importância da experiência desses profissionais para evitar novas despesas com reformas. Ainda, a autora, destaca, de forma descritiva, as rotinas e a estrutura física de um Departamento de Pediatria em um hospital geral, tais como: área física, ambiente, recreação, isolamento, limpeza, roupas, entre outros.

Ainda, para Veloso ${ }^{(20)}$, os hospitais infantis necessitavam de enfermeiras diplomadas com "certa especialização" em enfermagem pediátrica para orientar as demais, demonstrando que a enfermagem encontrava-se no caminho de desenvolverse no campo da especialização, acompanhando as especializações médicas na área de pediatria.

Por outro lado, Teixeira ${ }^{(21)}$ critica o preparo das enfermeiras nas escolas, demonstrando entre as dificuldades encontradas por estas profissionais o seu desconhecimento no lidar com o comportamento da criança e suas necessidade, conhecimentos sobre nutrição e dietética infantil e pouco interesse sobre a recreação e terapia ocupacional.

Oliveira(22) enfatiza a seleção de pessoal e o aprimoramento das enfermeiras para cuidar da criança hospitalizada, assim como o conhecimento sobre o 
desenvolvimento biopsicossocial da criança e o papel da mãe substituta.

\section{Considerações finais}

No início do século $X X$, o setor saúde deixa o caráter social e filantrópico para ser subsidiado pelo governo. Em relação a assistência à criança, inúmeros hospitais são inaugurados nos anos 30 e 40, apesar da política nacional de saúde caracterizar-se por ações preventivas, e persistir o movimento eugênico.

As enfermeiras reproduziam o discurso ideológicogovernamental em relação a taxa de mortalidade infantil, analfabetismo, condições sócio-econômicas, entre outros.

As transformações da política de saúde infantil e o fortalecimento das ações curativas, com o advento de novas tecnologias, propiciaram cada vez mais as ações de enfermagem no contexto hospitalar, resultando na necessidade de uma assistência diferenciada à criança.

Nos anos 50, o hospital é o centro de atendimento à saúde, e na área de pediatria, a assistência à criança concentrase em hospitais especializados ou enfermarias específicas nos hospitais gerais.

As enfermeiras destacavam a necessidade da aquisição de conhecimentos técnico-científicos para cuidar das crianças hospitalizadas em diferentes situações.

$A$ assistência de enfermagem à criança, na primeira metade do século XX caracteriza-se por transformações técnicocientíficas no cenário hospitalar, favorecendo a construção do saber da enfermagem na área de pediatria.

\section{Referências}

1. Oliveira ICS. (Re) construindo a assistência de enfermagem à criança hospitalizada na cidade do Rio de Janeiro (1920-1969) [tese doutorado em enfermagem]. Rio de Janeiro (RJ): Escola de Enfermagem Anna Nery, Universidade Federal do Rio de Janeiro; 1996.180f.

2. Orlandi OV. Teoria e prática do amor à criança: Introdução à pediatria social no Brasil. Rio de Janeiro: Jorge Zahar;1985.174 p.

3. Ribeiro L. Tempo de Médico (Almir Madeira e a assistência à infância). Rio de Janeiro:Cátedra;1979.145p.

4. Telles W. Um século de Pediatria. Jornal de Pediatria, Rio de Janeiro 1983;59(4):206-10.

5. Rodrigues BA. Fundamentos de Administração Sanitária. $2^{\mathrm{a}}$ ed Brasília(DF);1979.274p.

6. Paixão W. História da Enfermagem. $5^{a}$ ed. Rio de Janeiro: Julio Reis; 1979.138p.
7. Figueiredo G. Conferência Nacional de Proteção à Infância. Annaes de Enfermagem, Rio de Janeiro 1933 dez;1(2):35-38.

8. Forjaz MCS. Tenentismo e Forças Armadas na revolução de 30. Rio de Janeiro: Forense Universitária;1988.62p.

9. Braga JCS, Goes de Paula S. Saúde e Previdência: estudos de política social. São Paulo: Cebes/Hucitec;1981.226p.

10. Ferriani MGC, Rocha SMM, Cano MAT. A prática de enfermagem pediátrica no Estado de São Paulo. In: CBEn. Anais do $32^{\circ}$ Congresso Brasileiro de Enfermagem;1985 nov 17-22; Olinda (PE), Brasil. Olinda (PE): ABEn;1985.p.371-381.

11. Fonseca CMO. Modelando a cera virgem: a saúde da criança na política social de Vargas [dissertação de Mestrado em História]. Niterói (RJ): Instituto de Ciências Humanas e Filosofia, Universidade Federal Fluminense;1990.120p.

12. Secretaria de Saúde do Estado da Guanabara. Assistência Pública Guanabara - 80 anos de História Estado da Guanabara: Secretaria de Saúde;1972.

13. Instituto de Puericultura da Universidade do Brasil. Boletim do Instituto de Puericultura da Universidade do Brasil. Rio de Janeiro 1953; 10(25):8-35.

14. Alves CP. Aspecto social das doenças nas crianças. Annaes de Enfermagem Rio de Janeiro 1937 set; 10(5):40-41.

15. Figueiredo G. Maternidade, infância e adolescência. Rio de Janeiro: DNCr;1945.480p.

16. Bodstein RCA, coordenadora. História e saúde pública: A política de controle de câncer no Brasil. Rio de Janeiro: Pec/Ensp;1987.209p.

17. Marques AN. O exercício da puericultura na atualidade brasileira. Jornal de Pediatria: Rio de Janeiro 1990 jan/mar;66(1/3):33-40.

18. Donnangelo MCF. Medicina e Sociedade: o médico e seu mercado de trabalho. São Paulo: Pioneira;1975.176p.

19. Alvim EF. Reuniões de grupo para discussão dos problemas da criança pré-escolar. Anais de Enfermagem Rio de Janeiro 1954 mar;5(1):36-41.

20. Veloso N. Enfermagem Pediátrica: Mudança de atitude em relação à criança. Anais de Enfermagem Rio de Janeiro 1952 dez;1(2):167177.

21. Teixeira G. O que a enfermagem hospitalar exige da enfermeira. Revista Brasileira de Enfermagem Brasília (DF) 1958 dez;11(4):405409.

22. Oliveira GG. Assistência de enfermagem à criança. Revista Brasileira de Enfermagem, Rio de Janeiro 1964 out;27(5):318-24.

Data de Recebimento: 26/01/2003

Data de Aprovação: 30/04/2004 